\title{
LA RADIO DE TOUS LES MAUX
}

\author{
Blandine Schmidt ${ }^{1}$
}

À l'heure de la généralisation de la parole des anonymes dans les médias, cet article s'interroge sur l'évolution des modalités d'expression du moi et de ses difficultés personnelles dans le cadre radiophonique. Malgré la diversité des genres, des thématiques et des époques concernant les programmes interactifs diffusés sur les ondes, la plupart d'entre eux prennent le parti d'exposer la vie privée des personnes sur la place publique. L'assistance, le soutien voire la prise en charge de leurs problèmes sont couramment en toile de fond. Nous concentrerons plus particulièrement notre attention sur les dispositifs interactifs radiophoniques ainsi que sur la place accordée à la parole des auditeurs dans le cadre de l'émission de Menie Grégoire sur RTL de 1967 à 1981 et au sein de "Lahaie, l'amour et vous » diffusée sur RMC depuis 2001. Animées toutes les deux par des femmes, elles se proposent de recueillir sur un mode cathartique les témoignages intimes des personnes dans le besoin afin de les conseiller ou de les aider. En confrontant les différents dispositifs mis en place entre les années 1960 / 1970 et les années 2000, et leurs appropriations par les auditeurs, nous reviendrons sur leurs temps d'expression, leurs degrés d'implication et les différents modes discursifs afin de mieux saisir la portée et l'impact de la parole profane à la radio.

1 Blandine Schmidt est doctorante et ATER en Sciences de l'Information et de la Communication, Université Bordeaux III.

Recherches en communication, $\mathrm{n}^{\circ} 37$ (2012). 
Permettant d'établir un lien avec les autres, « la parole aujourd'hui est un fait social majeur. »(Breton, 2003, p. 285). Selon Philippe Breton, c'est par elle que nous passons pour débattre, pour témoigner, pour argumenter. C'est par elle que « nous organisons et transformons le monde qui nous entoure. » (Breton, 2003, p. 285). De nos jours, son déploiement est facilité par de nombreux moyens de communication. La radio, média de l'oralité par essence, lui accorde une place centrale. Dans un premier temps réservée aux experts, la parole est aujourd'hui donnée aux profanes, témoins et acteurs de la vie ordinaire. La véracité de leurs propos est rarement remise en cause dans la mesure où ils sont invités à fournir un témoignage. Celui-ci est considéré comme authentique étant basée sur une expérience personnelle. De plus, pour Patrick Charaudeau et Rodolphe Ghiglione, les sociétés occidentales ont attribué à la parole « le pouvoir de dire la vérité » (Charaudeau \& Ghiglione, 1997, p. 28). Selon eux, même si la parole se distingue de la vérité, elle constitue la condition pour l'atteindre en lui attribuant une grande valeur symbolique.

Le récit de soi, de son expérience, de ses difficultés personnelles dans les médias s'est largement développé au cours de ces quarante dernières années. Tendant à banaliser l'expression des anonymes sur la sphère publique, les radios françaises ont participé à ce phénomène par la mise en place de programmes interactifs. Ayant gagné les faveurs des professionnels, ils sont souvent dynamiques, colorent l'antenne par la variété des voix et des accents des personnes qui s'y expriment et garantissent de bonnes audiences. Malgré la diversité des genres et des thématiques, la plupart d'entre eux exposent la vie privée des auditeurs sur la place publique. L'assistance, le soutien voire la prise en charge de leurs problèmes sont couramment en toile de fond. Nous souhaitons ici revenir sur les modalités de prise de parole du public dans ce type de programme. Répondant aux sollicitations du média, les appelants osent parfois évoquer sur les ondes des choses tues dans le domaine privé. Prenant en compte cette dynamique de témoignages basés sur l'intime, quels sont les dispositifs et la place accordés à l'expression du moi dans l'espace radiophonique?

Afin d'apporter des éléments de réponses tout en offrant un éclairage sur l'évolution du récit de soi dans le cadre médiatique, nous présenterons le cas de deux émissions interactives aspirant à résoudre les problèmes rencontrés par des anonymes dans leur vie privée. La 
première fut animée par Menie Grégoire sur RTL dans les années 1960 - 1970 et la seconde intitulée Lahaie, l'amour et vous est diffusée depuis 2001 sur RMC-Info. Toutes deux se basent sur la participation à l'antenne des auditeurs, invités à se dévoiler sur un mode cathartique afin de recevoir le soutien et l'aide des animatrices. Après une présentation des ces émissions et de leurs déroulements, nous détaillerons les différents dispositifs interactifs mis à disposition des auditeurs pour favoriser leur expression sur les ondes.

\section{Présentation de l'émission de Menie Grégoire}

L'arrivée de Menie Grégoire sur RTL en mars 1967 bouleverse le paysage radiophonique français tant du point de vue du contenu diffusé que du dispositif communicationnel mis en place. Initialement, Menie Grégoire devait intervenir dans le cadre d'une émission intitulée Allô Luxembourg. Souhaitant exploiter intensivement un dispositif existant mais encore peu répandu, Jean Farran, le nouveau directeur de la station, veut faire participer les auditeurs à l'antenne par le biais du téléphone. Ces derniers pouvaient joindre la station pour poser leurs questions et un spécialiste devait leur fournir une réponse. Menie Grégoire avait la responsabilité des problèmes concernant la gent féminine. Alors qu'aucun appel ne lui est destiné, elle décide de prendre l'antenne en piochant dans l'abondant courrier reçu au magazine Elle suite à la publication de ses articles.

Un jour, j'ai pris mon courage à deux mains. J'avais un paquet de lettres extraordinaires où les femmes me disaient ce qu'on ne dit jamais, ce qu'on ne dit jamais dans les journaux non plus. Une histoire de sexe avec son mari. Alors, je suis arrivée. J'ai lu une lettre. J'ai répondu. Je suis rentrée chez moi. Le lendemain, il y avait 50 lettres. Donc, j'ai continué. (Entretien réalisé par l'auteur avec Menie Grégoire le 18 novembre 2008).

Rien ne pouvait présager à l'époque que l'engouement du public serait si grand et qu'un nouveau genre était en train de naître : la confession radiophonique. Quotidiennement et durant quatorze ans, Menie Grégoire a accueilli sur son antenne les témoignages d'auditeurs mais surtout d'auditrices dans le besoin venant chercher son réconfort concernant leur vie intime, familiale ou professionnelle. L'émission qui n'avait d'autre nom que celui de l'animatrice se déroulait de manière 
quasi similaire chaque jour. Sans préambule, la lettre d'un auditeur était lue en direct suivie d'une réponse personnelle de l'animatrice. Elle s'adressait directement à son expéditeur en le questionnant, en lui donnant son point de vue et quelques pistes de réflexion. Elle poursuivait en accueillant en direct deux à trois auditeurs durant six à neuf minutes chacun. La sélection opérée devait offrir une complémentarité dans les témoignages afin d'aborder les différents aspects de la thématique du jour. Laissant les auditeurs exposer leurs situations, elle les interrogeait d'une voix rassurante afin qu'ils précisent leurs récits et dévoilent leurs ressentis. Pendant ce temps, les assistantes à l'écoute communiquaient avec l'animatrice par des gestes et des petites notes écrites pour l'aider dans ses relances et dans l'orientation de la conversation. Les auditeurs pouvaient se plaindre et exposer leurs souffrances au grand jour. Menie Grégoire était là pour les rassurer et les réconforter même si elle pouvait quelque fois prendre un ton accusateur. « J'étais devenue la confidente, celle avec qui on a envie de parler. » (Entretien réalisé par l'auteur avec Menie Grégoire le 18 novembre 2008). Lorsque les auditeurs se confient à la radio, ils ne réalisent pas toujours que leurs conversations sont entendues par de très nombreuses personnes derrières leurs postes. Les émissions interactives radiophoniques jouent sur ce faux-semblant. Dans la mesure où les intervenants ne sont pas présents dans le studio, le dispositif ne diffère pas d'une conversation téléphonique privée. En fait, ils ne s'adressent pas aux auditeurs, ils sont là pour parler à Menie Grégoire. Le passage sur les ondes n'est que le moyen de parvenir à ce but. Cette émission a contribué à libérer la parole sur des sujets tabous en rompant la frontière entre la sphère privée et la sphère publique au sein d'une société qui vivait des mutations sociales importantes.

\section{Présentation de l'émission « Lahaie, l'amour et vous »}

Traitant d'amour, d'intimité et de sexualité, ce programme interactif est animé par Brigitte Lahaie du lundi au vendredi de 14 heures à 16 heures. À l'initiative de la nouvelle direction de RMC-Info, il a généré à ses débuts une certaine agitation médiatique revenant en particulier sur la carrière pornographique de l'animatrice. Afin de se prémunir de toute dérive et d'apaiser les craintes concernant la diffusion d'un programme destiné aux adultes en journée, une charte approuvée par le Conseil Supérieur de l'Audiovisuel a été rédigée par la station pour définir les valeurs et principes de ce rendez-vous radiophonique. 
Il est ainsi précisé que " l'émission du mercredi [jour de repos dans les écoles françaises] s'adresse à un public plus familial où la sexualité des adolescents est abordée pour répondre à leurs questions et à celles de leurs parents. » (CSA, Rapport d'activité 2004, 3 octobre 2005). Toutefois, nous avons pu constater que celle-ci se concentre plus sur des thèmes liés à l'amour que sur ceux liés à la sexualité. Que ce soit sur le site internet de RMC-Info (http://www.rmc.fr/) ou sur le blog de l'animatrice (http://www.rmc.fr/blogs/brigittelahaie.php), les auditeurs peuvent retrouver les informations développées à l'antenne, des contenus enrichis, des services complémentaires tels que le podcast ou encore des interfaces interactives comme le forum. Adoptant une stratégie de cross-média, "Lahaie, l'amour et vous " se prolonge ainsi sur le web. Réalisée en direct chaque jour de la semaine, à l'exception du vendredi où elle est préenregistrée en faux direct, l'émission est divisée en six modules qui sont encadrés par des annonces publicitaires. S'agissant d'une radio privée et commerciale, l'antenne est plus organisée en fonction des espaces publicitaires qu'en fonction des contenus.

Introduite par un jingle musical moderne et dynamique, l'animatrice va brièvement annoncer le sommaire, exposer la thématique du jour et présenter son invité expert venant la soutenir et compléter ses réponses. Elle enchaîne rapidement en accueillant un premier auditeur à l'antenne. Malgré la définition d'une thématique quotidienne, toutes les interventions ne s'y rapportent pas, permettant une plus grande marge de manœuvre tout en diversifiant les témoignages. En moyenne, une douzaine d'auditeurs se succèdent à l'antenne pour parler de leurs difficultés d'ordre physique, psychologique ou relationnel. N'hésitant pas à entrer dans les détails, ils s'adressent à l'animatrice comme à une confidente et sollicitent son expertise. Cette dernière leur fournit une écoute attentive afin de favoriser une libre expression. Sur un ton amical, elle les questionne et leur fait développer leur récit. Venant chercher du réconfort mais également un conseil personnalisé de Brigitte Lahaie, ils ont confiance en son jugement. Certains considèrent même que seule l'animatrice saura trouver la solution. S'il s'agit d'un problème de sexualité, l'intervention sera plus courte (de deux à quatre minutes) que s'il s'agit d'un problème d'ordre psychologique (de cinq à dix minutes). Toutefois, l'animatrice affirme ne pas tenir compte du critère temporel dans ses échanges : "Quand je suis avec un auditeur, je ne regarde absolument pas à quelle minute je le prends et à quelle minute je le rends. [...] Ce n'est vraiment pas dans mon éthique.» (Entretien réalisé 
par l'auteur avec Brigitte Lahaie le 18 juin 2009). Plusieurs rubriques sont également proposées pour rythmer le programme. Par exemple, la rubrique « J'ai testé pour vous » met en scène un auditeur ayant expérimenté un jouet sexuel que la station lui a précédemment fait parvenir. Il va le décrire, donner ses impressions et l'évaluer en lui attribuant une note. Enfin, l'émission est complétée par l'interview de l'invité et de différentes rubriques à visées pédagogiques.

\section{Le récit de soi dans le cadre médiatique}

\section{Dans l'émission de Menie Grégoire}

S'agissant du début des émissions interactives, les auditeurs de Menie Grégoire n'ont que deux possibilités pour entrer en contact avec la station : l'envoi d'une lettre manuscrite ou l'usage du téléphone.

\section{Le courrier des auditeurs}

La rédaction d'une lettre à un média n'était pas un fait nouveau pour le public français. Existant depuis de nombreuses années, le courrier des lecteurs constituait une rubrique à part entière dans les magazines féminins. C'est d'ailleurs au sein de ce type de publication que Menie Grégoire a débuté sa carrière. À la radio, ces correspondances ont acquis une dimension plus vivante grâce à leurs passages à l'oral. Le processus communicationnel est également enrichi par la réponse fournie aux personnes. L'animatrice a reçu de très nombreuses lettres écrites spontanément ou en réaction à ce qui se disait à l'antenne. En effet, l'engagement de l'auditoire dans ce type de programme est important. On s'identifie et on se compare avec ceux qui interviennent sur les ondes. Il devient ainsi nécessaire pour les écoutants de confier leur propre expérience et de donner leur avis. Les lettres de réaction permettaient ainsi d'alimenter les émissions suivantes.

Pour la lecture qui allait en être faite à l'antenne, l'animatrice effectuait de nombreuses coupes dans les lettres longues de plusieurs pages. Ceci permettait de garantir une certaine clarté tout en préservant l'anonymat de l'expéditeur. Les nombreux détails présents font transparaître le besoin des personnes de se confier en disant tout ce qu'elles ont sur le cœur. Proches des confessions que l'on peut faire à un journal intime, 
ces lettres illustrent le grand désespoir des gens n'ayant personne d'autre à qui raconter leur histoire. Permettant de ne pas faire face à quelqu'un et de ne pas affronter son regard, l'acte d'écriture n'est pas dénué de sens. Lorsque l'on écrit une lettre, on ne s'adresse pas seulement à l'autre, on écrit aussi à soi-même. De plus, il est nécessaire d'organiser ses pensées afin d'être le plus clair possible. Se confier à quelqu'un permet également de rompre la solitude dans laquelle le problème nous plonge. Ainsi, écrire une lettre à Menie Grégoire était un acte libérateur. Les auditeurs sollicitaient une personne qui ne les connaissait pas personnellement mais qui était proche d'eux grâce à la quotidienneté de l'émission. « J'étais Menie Grégoire, c'est à dire quelqu'un qui devait comprendre et qui devait aider à comprendre. » (Entretien réalisé par l'auteur avec Menie Grégoire le 18 novembre 2008).

Quotidiennement, l'animatrice recevait en moyenne une centaine de lettres. Si le sujet traité à l'antenne était particulièrement brûlant, elle pouvait en recevoir jusqu'à six cents. Le travail statistique de Dominique Cardon et Smaïn Laacher (Cité en Robert, 1998, p. 106) nous aiguille sur l'évolution des grands thèmes évoqués par les auditeurs. Lors des premières années d'existence du programme, les problèmes liés au couple et à la famille tenaient une place prépondérante. Les lettres traitant de sexualité, de psychologie et des difficultés rencontrées dans la vie quotidienne vont progressivement prendre de l'ampleur au fil des années 1970 .

Le traitement du courrier était une lourde tâche confiée aux assistantes de l'animatrice. L'une d'entre elles, Janine Blanchard, raconte : « J'avais des masses de lettres, des centaines de lettres, il fallait que je résume en quelques mots sur le dos de l'enveloppe. J'en lisais et j’en résumais une centaine par jour. » (Cité en Robert, 1998, p. 101). Comme l'explique Menie Grégoire, elles étaient également en charge de leur sélection en vue d'un passage à l'antenne :

Mes assistantes sélectionnaient les lettres qui méritaient de lancer un sujet particulièrement émouvant ou passionnant. $\mathrm{Ce}$ sont elles qui savaient ce qui était dans le vent. On dressait des courbes sur le mur sur la fréquence des thèmes. On prenait la lettre parce qu'elle était intéressante ou parce qu'il y avait quelque chose de neuf et qu'elle tombait dans les choses qui intéressaient à ce moment là ou bien parce que c'était une belle lettre intelligente. Les critères, on ne les a jamais mis noir sur 
blanc. (Entretien réalisé par l'auteur avec Menie Grégoire le 18 novembre 2008).

\section{Les appels téléphoniques}

Le pari initial de Jean Farran de faire intervenir des auditeurs à l'antenne par le biais du téléphone n'était pas gagné d'avance. En effet, en comparaison à d'autres pays occidentaux, la France a subi un lourd retard concernant l'équipement des foyers. Ceci pouvait générer de nombreuses difficultés pour passer un coup de téléphone comme l'illustre le célèbre sketch de Fernand Raynaud Le 22 à Asnières. Longtemps considéré comme un outil de luxe, le téléphone va progressivement gagner du terrain en France. De 1950 à 1967, l'équipement a doublé, passant d'un million et demi de lignes principales à plus de trois millions. Ceci demeurait toutefois assez faible avec un taux d'équipement de 6,4 pour 100 habitants (Robert, 1998, p. 97). Cette carence était en partie compensée par l'existence de cabines publiques qui toutefois, ne permettaient pas une grande intimité comme le rapporte Menie Grégoire : « Le téléphone, tout le monde ne l'avait pas. Alors on allait à la Poste. Mais on devait changer de bureau de Poste parce qu'il y avait la demoiselle des Postes qui vous connaissait... » (Entretien réalisé par l'auteur avec Menie Grégoire le 18 novembre 2008). Dès le début de l'émission, les appels provenant de la France entière arrivaient au standard de RTL. Les standardistes, souvent des jeunes entre deux travails, synthétisaient en quelques phrases l'histoire de la personne sur deux ou trois pages. Même si elle ne souhaitait pas intervenir à l'antenne, une fiche était systématiquement réalisée afin de connaître les motivations de son appel. Ensuite, les assistantes prenaient le relai pour la sélection finale en se basant sur l'intérêt du cas exposé mais aussi de l'élocution et l'esthétique de la voix.

Le dialogue entretenu entre Menie Grégoire et ses auditeurs a souvent été qualifié de confession. L'analogie avec cette pratique religieuse est justifiée par la nature des interventions des appelants et par les conditions imposées par le dispositif technique. En effet, lorsque l'on s'adresse à Menie Grégoire par le biais du téléphone, on ne la voit pas, on est dans le noir comme dans un confessionnal. On peut tout dire, se débarrasser de son fardeau de manière anonyme sans avoir à subir le regard accusateur de son interlocuteur. À l'église, le prêtre va écouter, questionner et absoudre le pénitent. Toutefois, alors que la reli- 
gion propose un code de conduite pour définir ce qui est bien ou mal, Menie Grégoire ne jugeait pas ses auditeurs. Préférant adopter un ton compassionnel et rassurant, elle les guidait dans leurs introspections.

\section{Dans l'émission « Lahaie, l'amour et vous »}

RMC-Info ciblant l'essentiel de ses programmes sur la participation du public, de nombreux dispositifs sont mis en place pour stimuler la relation interactive.

\section{Les appels téléphoniques}

Les appels reçus constituent une ressource financière complémentaire pour la station qui ne met à disposition des auditeurs qu'un numéro surtaxé. Ne retenant que ceux qui souhaitent intervenir à l'antenne, le standardiste créée quotidiennement entre vingt et trente fiches électroniques qui reprennent le plus fidèlement possible les propos des auditeurs. Elles sont ensuite utilisées par le coordinateur du standard pour réaliser le casting, c'est à dire la sélection finale. Il rappelle les cas qu'il juge les plus intéressants en ciblant prioritairement les jeunes femmes. L'auditorat de RMC-Info étant composé en grande majorité d'hommes, il souhaite par ce biais créer une ambiance fantasmagorique quitte à véhiculer un standard hétérosexuel. Après avoir détaillé son cas au coordinateur, l'auditeur, s'il est sélectionné, est mis en attente puis basculé sur l'antenne afin de dialoguer avec l'animatrice. À la fin de son intervention, le coordinateur va évaluer son comportement à l'antenne et lui attribuer une note qui sera reportée sur sa fiche. Il consultera cette dernière si la personne souhaite intervenir de nouveau sur RMC-Info. Les SMS ne sont pas utilisés dans le cadre de cette émission même s'il est possible d'en envoyer à la station. Enfin, l'équipe de Brigitte Lahaie détourne l'usage du répondeur téléphonique par la mise en scène d'un auditeur fictif en préenregistrant un message au contenu plus osé que la normale.

\section{Les messages électroniques}

Accessible depuis la page web de « Lahaie, l'amour et vous », un formulaire permet aux auditeurs d'envoyer des messages électroniques à l'animatrice. Elle en reçoit une vingtaine par jour, le plus couramment postés durant l'émission ou durant sa rediffusion nocturne. Ceci indique 
qu'ils sont écrits à chaud en réaction à ce qui se dit à l'antenne. Sur la base des messages recueillis durant mon terrain de recherche en 2009, il est possible de constater que la plupart des internautes conservent leur anonymat. En effet, 92,5 \% d'entre eux communiquent leurs prénoms alors que $57 \%$ donnent leurs noms de famille. De plus, nous avons pu constater que les personnes laissent rarement leur numéro de téléphone (37\% des messages), manifestant leur souhait de ne pas intervenir en direct. Deux ou trois courriers électroniques sont quotidiennement sélectionnés par l'animatrice en fonction de leur originalité et de leur clarté pour être traité durant l'émission. Une réponse personnalisée va dans ce cas être fournie sans pour autant entrer dans les détails afin de s'adapter aux attentes de la globalité de l'auditoire. Les émissions interactives jouent sur l'idée qu'une réponse apportée à une personne en particulier va pouvoir en aider d'autres vivant une expérience similaire. Ceci fait référence au principe de la «main invisible» de l'économiste Adam Smith. Ce dernier considère que lorsqu'un individu vise la satisfaction d'un intérêt personnel, cela va favoriser l'intérêt général. Selon Michel Meyer, il s'agit d'une " loi qui s'adapte de fait aux émissions interactives en ce sens qu'un auditeur appelant par intérêt personnel œuvre largement, pour ne pas dire à tous les coups, en faveur de l'intérêt général. Ainsi, en répondant aux attentes d'un seul auditeur, une émission interactive apporte une réponse générale à la multitude. » (Meyer, 2003, p. 190). Même s'il s'agit de l'histoire d'une personne en particulier, certains écoutants peuvent se reconnaître et s'identifier à la situation. Ils vont pouvoir s'approprier les conseils prodigués à l'antenne, amplifiant la portée de cette parole. Par ce biais, la radio va pouvoir rassembler ses auditeurs. Les messages reçus vont être plus généralement utilisés dans la préparation de l'émission. Brigitte Lahaie prend en compte la récurrence des sujets qui y sont évoqués pour définir la thématique quotidienne. Hormis quelques exceptions, la plupart d'entre eux sont courts, concis et rédigés dans un style familier proche du langage parlé. Il est possible de distinguer différents types de messages en fonction de leurs contenus. Il y a tout d'abord les témoignages couplés à des questions très précises. Le récit de quelques lignes est une mise en contexte du problème de l'auditeur. Ces messages sont les plus récurrents et constituent la moitié du courrier reçu quotidiennement. Suivent les demandes de conseils, souvent de nature psychologique, directement adressées à l'animatrice. D'autres sont envoyés à l'intention d'auditeurs intervenus à l'antenne. Ayant eux-mêmes vécu une situation similaire, les expéditeurs souhaitent partager leurs propres 
expériences, les conseiller, voire être mis directement en contact avec eux. Ceci révèle l'existence d'une relation de solidarité entre les auditeurs. D'autres encore veulent obtenir des renseignements (livres, sites internet) ou des contacts de professionnels correspondant à la dimension pédagogique de ce programme. L'animatrice reçoit également des propositions de sujet ou des demandes de participations à certaines rubriques. Enfin, il y a les traditionnels messages de félicitations qui complimentent son émission ou directement elle-même, rédigés sur le ton de la séduction.

\section{Les sondages en ligne}

Depuis la rentrée 2010, de nouveaux sondages sont apparus sur le site web de RMC-Info. Le nombre de participants est variable, allant de plusieurs centaines de votants à plus d'un millier. Existant depuis les débuts des années 2000, ils trouvent désormais un écho à l'antenne. Par exemple, « Lahaie, l'amour et vous » propose un sondage en lien direct avec la thématique du jour. Accessible dès le matin de l'émission, les questions viennent compléter les interventions des auditeurs en direct et portent le plus souvent sur leurs pratiques sexuelles : « Avezvous déjà eu une expérience à trois ? », « Combien de fois par semaine faites-vous l'amour? », «Avez-vous déjà eu recours aux services d'une prostituée ? ». La productrice de l'émission intervient à l'antenne pour dévoiler les résultats qui seront par la suite commentés par l'animatrice. Autre exemple : « Le sondage sexy de la semaine » qui se concentre tantôt sur la sexualité au sens large tantôt sur le contenu du programme permettant d'aiguiller l'équipe sur les attentes des auditeurs sondés.

\section{Le forum en ligne}

Contrairement aux autres forums de RMC-Info, il est nécessaire d'être majeur pour accéder à celui de "Lahaie, l'amour et vous » dans la mesure où certains contenus peuvent choquer le jeune public même si aucun contenu pornographique ou illégal n'est autorisé à la publication. Il est possible de poster des messages au sein de différentes rubriques créées par la modératrice ou par les internautes. Ces derniers peuvent venir alimenter la discussion engagée dans cet espace ou répondre plus particulièrement au message d'une personne en mode privé. Ils s'expriment de manière décontractée, proche du langage parlé et l'utilisation d'émoticônes en guise de ponctuation est très courante 
dans leurs messages. Durant l'émission, la modératrice interviendra à l'antenne pour inciter les auditeurs à se connecter mais également pour donner un aperçu sur les ondes de ce qui se dit en ligne. Le forum peut donc constituer une passerelle vers l'antenne même si les personnes ne s'y expriment pas directement.

\section{Conclusion}

Comme nous l'avons montré, le récit de soi dans le cadre médiatique s'est développé au fil des années. D'abord, le temps accordé à l'expression des auditeurs a augmenté : alors que l'émission de Menie Grégoire ne durait qu'une demi-heure, celle de Brigitte Lahaie s'étend sur deux heures. Puis les dispositifs interactifs se sont multipliés, soutenus par le développement des nouvelles technologies de communication et leur appropriation par le public. Selon leurs degrés d'implication, les auditeurs peuvent choisir de quelle manière ils participeront à l'émission ; l'important étant qu'ils soient actifs et réactifs pour fidéliser leur écoute. Une autre stratégie permettant au média de réunir son public a vu le jour grâce à l'extension du programme sur le site web de la station. Alors que les auditeurs de Menie Grégoire ne s'adressaient qu'à elle, ceux de Brigitte Lahaie ont la possibilité de se rencontrer virtuellement sur le forum et de dialoguer publiquement ou en mode privé. Ce prolongement de l'émission favorise la création d'une communauté d'auditeurs.

Préférant l'envoi d'un courrier électronique à la rédaction d'une lettre manuscrite, les modes discursifs ont également évolué depuis les années 1960/1970. Alors que l'on se confiait sur des pages entières à Menie Grégoire, les messages envoyés à Brigitte Lahaie sont courts et exposent sans ambages le problème dont il est question. Ceci montre aussi que les auditeurs se sont familiarisés avec les dispositifs interactifs et qu'ils ont conscience de la participation que l'on attend d'eux. Enfin, le dévoilement de soi est aujourd'hui plus important, soutenu par la diversification des thématiques évoquées à l'antenne.

Vingt ans séparent les émissions de Menie Grégoire et de Brigitte Lahaie. L'évolution des mœurs ainsi que la généralisation du récit de l'intime dans les médias ont facilité l'acceptation de ce type de programmes par le public français. En effet, Menie Grégoire a dû faire face à de nombreuses critiques provenant du monde médical et des instances médiatiques qui l'accusaient d'être une « briseuse de 
ménage » (Ici Paris, 25 décembre 1972) ou qualifiant son émission de « radio sans culotte » (L'Aurore, 7 novembre 1973). Même si certains peuvent reprocher à Brigitte Lahaie un manque de décence, les avis lui sont plutôt favorables : «Que 1'on évoque la technique sexuelle ou les sentiments, 1'ex-star du X a le même ton respectueux et bienveillant. » (Le Monde, 22 novembre 2009). Aujourd'hui la rupture entre la sphère publique et la sphère privée est un phénomène acquis et accepté par le public. L'expression de soi s'étant banalisée dans les médias, les personnes exposent leur intimité au grand jour sans pudeur. Le récit de soi à la radio devient une marque de reconnaissance pour l'individu qui aura par ce biais la possibilité de sortir de la masse. Au détriment de sa vie privée, il espère tirer un certain bénéfice de son passage à l'antenne.

\section{Références}

Breton, P. (2003). Éloge de la parole. Paris : La découverte.

Charaudeau, P., \& Ghiglione, R. (1997). La parole confisquée. Paris : Dunod.

Meyer, M. (2003). Paroles d'auditeurs. Paris : Syrtes.

Robert, G. (1998). Le divan radiophonique de Menie Grégoire. Cahiers d'Histoire de la Radiodiffusion, 55, pp. 88-154. 